\title{
La contribución de Amir Smith Córdoba a la infraestructura intelectual negra colombiana en los años setenta: aprender a ser negro con el periódico Presencia Negra (1979) ${ }^{1}$
}

\author{
Contribution of Amir Smith Córdoba to the Colombian
} BLACK INTELLECTUAL INFRASTRUCTURE IN THE I97OS: LEARNING TO Be Black with the newspaper PRESENCIA NEGRA (1979)

\author{
Silvia Valero \\ Universidad de Cartagena, Cartagena, Colombia \\ svalero@unicartagena.edu.co \\ Richard Delgado \\ Universidad de Cartagena, Cartagena, Colombia \\ rdelgador@unicartagena.edu.co
}

Resumen: Durante la década de los setenta, Amir Smith Córdoba funda el Centro para la Investigación de la Cultura Negra (CIDCUN), el cual, junto con su periódico Presencia Negra, fue parte de la plataforma de lucha

\footnotetext{
Este trabajo es producto del proyecto de investigación "Entre el particularismo y el universalismo: tensiones discursivas en tres publicaciones seriadas de Colombia en torno a las negritudes (1975-1980)", financiado por la Vicerrectoría de Investigaciones de la Universidad de Cartagena (Res. 02005/2018). En razón de que este proyecto se concentra en la década del setenta, nos enfocaremos en los números de Presencia Negra publicados en 1979.
} 
por esa época en Colombia, conformando lo que denominamos en este trabajo la "infraestructura intelectual negra". El objetivo de este artículo es explorar, en los primeros números del periódico Presencia Negra, la producción del proyecto normativo/pedagogizador de Smith Córdoba, que se desarrolló tanto alrededor de una retórica del "deber ser negro", como a través de columnas-respuestas que buscaron "desalienar" también a cierto sector del periodismo, denominado por el autor-siguiendo a Césaire-como "pequeño burgués". En resumen, se buscará descifrar el significado de esa liberación cognitiva o desalienación, para Amir Smith Córdoba, y de qué recursos se valió para impulsarla.

Palabras Clave: infraestructura intelectual negra colombiana, Amir Smith Córdoba, CIDCUN, negritud, Presencia Negra.

Авstract: During the 1970s, Amir Smith Córdoba founds the Center for Research on Black Culture (CIDCUN). This Center and its newspaper Presencia Negra were part of the what we call in this work "Black Intellectual Infrastructure". The objective of this article is to explore, in the first issues of the newspaper Presencia Negra, the production of Smith Córdoba's normative / pedagogical project, which was developed both around a rhetoric of "must be black", and through articles that also sought to "de-alienate" a certain sector of journalism, called by the author, following Césaire, "petty bourgeois". In summary, it will seek to expose what this cognitive liberation or desalienation meant for Amir Smith Córdoba, and what resources he used to promote it.

Keywords: Colombian Black Intellectual Infrastructure, Amir Smith Córdoba, blackness, CIDCUN, Presencia Negra.

\section{INTRODUCCIÓN}

En medio de un dinámico escenario tanto político como intelectual en torno a las negritudes, que "despertó" -por utilizar el término de Gutiérrez Azopardo- en los años setenta en Colombia, el joven sociólogo Amir Smith Córdoba (Cértegui, 1948-Bogotá, 2003) fundó en 1975 el Centro para la Investigación de la Cultura Negra (CIDCUN) en Bogotá. Esta ciudad albergaba a numerosos estudiantes provenientes del Chocó, generando una activa vida intelectual y militante en el movimiento negro colombiano. Varios de estos estudiantes harán parte luego de la revista 
Negritud y el periódico Presencia Negra, órganos del CIDCUN, que alimentaron lo que llamamos la "infraestructura intelectual negra" de esos años ${ }^{2}$. Con "infraestructura intelectual negra" nos referimos a las organizaciones, espacios de estudio e investigación, publicaciones, y todas aquellas plataformas que, nucleadas por intelectuales negros -aunque también no negros-, sirvieron como plataformas para la producción de conocimiento y la gestión de programas de trabajo, cuyo objetivo fue revisar la historia de los ciudadanos negros colombianos, pero también actuar sobre su presente y proponer sobre su futuro.

El interés que despertó en los últimos años la conformación de ese campo intelectual negro de la década del setenta en Colombia -ejemplificado por los estudios de Peter Wade, como "Defining Blackness...", o los de Arboleda, Pisano, Valero, Valderrama, Munar y Valero y Ramos-, permitió que los proyectos institucionales y periodísticos de Smith Córdoba comenzaran a recibir mayor atención por parte de los investigadores. En poco tiempo, se incorporaron al ámbito académico artículos como "Reivindicación de la 'cultura negra' y la denuncia de discriminación racial en la experiencia del Centro de Estudios Afrocolombianos y el Centro para la Investigación de la Cultura Negra (CIDCUN)" -escrito por Lery Munar y publicado en 2020-, que es un agudo trabajo comparativo sobre los puntos de convergencia y disenso entre el CEA y el CIDCUN, partiendo de la concepción de ambos grupos de intelectuales negros como un estrato con autoconciencia racial; "La diferencia cultural negra en Colombia. Contrapúblicos afrocolombianos" - de Carlos Valderrama, aparecido en 2019-, el cual muestra el intercambio reticular del pensamiento y la movilización negra en la década del setenta; "Amir Smith Córdoba y la revista Negritud (1976): contrapuntos por el lugar del negro en la historia" - por Silvia Valero y Erick Ramos, publicado en 2020-, trabajo concentrado en el análisis del binomio historia/ negritud y la instrumentalización de la historia con fines políticos por parte de Smith Córdoba; "El racismo en Colombia: la visión de Amir Smith” -escrito por Jorge García Rincón y aparecido durante 2019-, que toma como fuente el libro Cultura Negra y avasallamiento cultural (1980) del chocoano, para analizar la importancia que tuvo la educación y la cultura en su proyecto de desalienación; y las tesis de maestría Amir

2 El denominador negro es usado en este trabajo para referir a la gente de ascendencia africana, respetando la terminología usada por Amir Smith Córdoba y los intelectuales de la época. 
Smith Córdoba. Del blanqueamiento a la negritud -escrita por Julio Arévalo en 2015- y Apprendre à être noir dans une nation métisse. La revue Presencia Negra et les mouvements noirs en Colombie dans les années 1980 -de Laura Martín Agudelo, investigación de 2019-.

La creación del CIDCUN representó la institucionalización de un espacio que tendría "el estudio de las causas y consecuencias de la discriminación racial hacia la gente negra como una prioridad en sus agendas de trabajo" (Munar 92. Cursivas del original) y serviría, también, como plataforma de lanzamiento de las dos publicaciones seriadas -Negritud y Presencia Negra- que el chocoano llevaría adelante como editor y redactor. Ambas se constituyen hoy en una vía de acceso para estudiar al intelectual y su práctica, pues tal como señala Pita González, el estudio integral de las publicaciones periódicas "enriquec[e] las perspectivas de la historia política, social, cultural e intelectual"3 (s/p).

Negritud y Presencia Negra nacieron en medio de un contexto con importantes/iniciales estudios en torno a las comunidades negras en Colombia, de corte antropológico y sociológico, que llegaron a dar nuevas luces sobre el estado de las negritudes en todo el territorio nacional, en un momento en que se producía "la consolidación del Palenque de San Basilio como símbolo iconográfico de resistencia” (Arboleda 52). El disparador de los estudios sobre Palenque fue el libro de Roberto Arrazola Caicedo, Palenque, primer pueblo libre de América, de 1970, aunque la consolidación se logró con los trabajos posteriores de Nina S. de Friedemann y Richard Cross, en 1979, y las investigaciones de Germán de Granday Carlos Patińo Roselli, ambas de 1978. Estos últimos trataban sobre la particularidad lingüística del Palenque de San Basilio (Arboleda 53-54). Estos estudios pioneros, que permitieron vislumbrar un importante campo científico desde lo local, fueron conocidos por Smith Córdoba, quien hizo visibles referencias a Palenque y a Benkos Biohó como ejemplos de la lucha negra en el país.

También la región del Pacífico colombiano fue objeto de estudios en los años setenta, como el de Aquiles Escalante, La minería del hambre: Condoto y la Chocó Pacifico, de 1971; y los de Norman Whitten y Nina S. de Friedemann, que publicaron investigaciones como "La cultura negra

3 Si bien Pita González se concentra en las revistas culturales, entendemos que la perspectiva que entiende el estudio actual de esas publicaciones como una herramienta de la historia intelectual es aplicable también a los periódicos. 
del litoral ecuatoriano y colombiano: un modelo de adaptación étnica", en 1974. Estos estudios seguramente fueron conocidos y seguidos de cerca por Amir Smith Córdoba, cuya preocupación por las condiciones de desigualdad y el abandono del Estado en su departamento de origen fueron objeto de análisis en su columna “¡Y para el Chocó qué?" (Smith Córdoba 1-5).

Motivado, entonces, por aquellos avances en el conocimiento, Smith Córdoba abordó los problemas económicos, sociales, artísticos, educativos y particularmente epistemológicos de la población negra de Colombia. Este intelectual entendía que dichos problemas tenían como vehículo principal a la raza, factor determinante de la alienación, la discriminación y la marginalización de las comunidades negras en un país sostenido por la ideología dominante del mestizaje.

La puesta en marcha de Presencia Negra a fines de los ańos setenta en Colombia, habla de la necesidad de los grupos de intelectuales que se conformaron en esta década de abrir espacios de discusión que escaparan a los recursos meramente académicos -lo que no implicaba que se negaran a ellos-y llegar, así, a la comunidad en general. Aunque Smith Córdoba había tenido su primera incursión en publicaciones de este tipo a través de la revista Negritud $^{4}$. El periódico seguramente resultó un soporte más fácil de sostener económicamente y prolongar en el tiempo que la revista, que contó con solo tres números. De tal manera, el diario podría convertirse en un facilitador para la resolución de lo problemático que significaba el acceso a espacios del mainstream periodístico manejados por una élite blanco-mestiza, en los cuales se desestimaba el problema del racismo en el ámbito nacional. Pero, aun más que eso, crear una plataforma propia para el desarrollo de un programa social e ideológico desde una perspectiva de desigualdad racial fue también una manera, para Smith Córdoba, de posicionarse en el campo intelectual negro de la época y reproducirse a sí mismo como intelectual'.

4 "El año de publicación de la revista tiene sus controversias: si bien en la tapa de los tres números publicados figura el año 1976, un estudio atento de su contenido demuestra que posiblemente no vieron la luz hasta 1978" (Valero y Ramos 148, cita 2).

5 Un punto relevante a conocer sería la capacidad de recepción que tuvo el periódico durante los años que vio la luz, así como su permanencia en el imaginario de los intelectuales negros a través del tiempo. Escapa a las posibilidades de este artículo una exploración de esa naturaleza, trabajo que queda pendiente para 
Los nombres con los que se bautizó a las publicaciones fueron elocuentes respecto del campo programático al que se circunscribirían y los modelos de los que se partía: en el caso de la revista, ello estaría sostenido por la categoría négritude, controvertida en la época (Valero y Ramos $149-156)^{6}$, pero que refería a un marcador de particularidad histórica y cultural y que, tal como se lo habían planteado sus creadores francófonos, convergía en la preocupación más hondamente articulada por Smith Córdoba, que era la urgencia de una concientización de los mismos negros acerca de su propia identidad, como veremos más adelante. Por el lado del periódico, Presencia Negra nos remonta directamente a la revista Présence Africaine, órgano difusor de la ideología de la négritude, fundado por Alioune Diop en 1947, como se manifiesta en el periódico mismo (Realpe Borja 5). En el caso colombiano, el título corporiza la presencia de la voz negra a través de la materialidad periodística. Ambas publicaciones del CIDCUN, en todo caso, expresaron la influencia de los creadores de la négritude en el pensamiento de Smith Córdoba.

Pero, además, y sobre todo desde la perspectiva de liberación del pensamiento del sujeto negro y sobre él, el fundamento teórico que más impactó en el chocoano, como en los intelectuales negros de la época en general, fue el de Frantz Fanon -con obras como Los condenados de la tierra - y su concepción de la alienación como proceso desestabilizador de la libertad intelectual, identitaria y ciudadana, no solo del sujeto negro sino también de cierto sector de la población blanco-mestiza. Desde allí que la búsqueda de la línea editorial de Presencia Negra fuera la desestigmatización de la negritud, como una continuidad ideológica de la revista Negritud, en tanto prolongaba el proyecto que se había visto truncado con la desaparición de esta última por las dificultades para su sostenimiento. Ahora bien, ¿qué significaba aquella liberación cognitiva o desalienación para Amir Smith Córdoba? ¿De qué recursos se valió para impulsarla?

futuras investigaciones. Un breve pero interesante acercamiento al tema se puede rastrear en el trabajo de tesis Apprendre à être noir dans une nation métisse. La revue Presencia Negra et les mouvements noirs en Colombie dans les années 1980, de Laura Martín Agudelo.

6 Una larga discusión con respecto a la adopción o no del término como representativo de un colectivo negro se desarrolló durante los debates del Primer Congreso de la Cultura Negra de las Américas (Valero 215-225). 
Argumentamos que, en Presencia Negra, Smith Córdoba apostó por un proyecto formador a través de la columna "Aprender a ser Negro", que necesariamente acogía una contraparte que era el "enseñar a ser". Por medio de ella y otros artículos, pugnó por un sujeto negro consciente de sí, también en la línea de Fanon -en Piel negra, máscaras blancas-, que chocaba radicalmente con lo trazado con anterioridad por la tradición del pensamiento occidental. Para ello, desarrolló un proyecto de pedagogización con el objetivo de que ese nuevo sujeto negro fuera partícipe consciente de la construcción del país. Participación y construcción que, según Smith Córdoba, solo podían darse por la vía política, es decir, que sujetos negros, educados en su autoconciencia, reclamaran los derechos que históricamente se les habían negado como ciudadanos. Sin embargo, el chocoano no encontraba que su proyecto intelectual tomase forma acabada sin el otro factor ciudadano que era la población blanco-mestiza colombiana. Esto significaba que, para una transformación social real, el proceso de desalienación debía desarmar la base epistemológica que sostenía el pensamiento social racializador de las clases dominantes. Entendemos que, aunque su mirada se extendía al imaginario en el orden general de la nación, en sus trabajos, Smith Córdoba se focalizó particularmente en los periodistas propagadores de prejuicios antinegro, pues sus espacios comunicativos les permitían el poder de producir sentido común, camino directo a la reificación de pensamientos.

\section{Aprender a ser negro: eje misional de PresenCIa Negra}

No es casual que, desde el primer número de Presencia Negra, el espacio reservado para "Aprender a ser Negro" sea la página inicial, pues esto explica, desde una perspectiva filosófica, que la columna se concibió como pilar del proyecto de "desavasallamiento" cultural impulsado por Smith Córdoba. Desde un punto de vista estructural, evidencia

El concepto "avasallamiento cultural” es usado por Smith Córdoba a lo largo de sus escritos y hará parte del título de su libro publicado en 1980, Cultura Negray avasallamiento cultural. Tomamos el término "desavasallamiento" de Valderrama y lo usamos aquí como indicador del objetivo del proyecto de Smith Córdoba, que, en líneas generales, apuntaba a "desavasallar", esto es, ir a contravía como manera de quebrar todo proceso de imposición cultural alienador de la población negra. 
que con ello se buscaba provocar un impacto visual movilizador en el lector, reforzado por el hecho de que cada columna fuera enmarcada por fotografías de personajes negros relevantes en el ámbito político, cultural y deportivo de la época. Estas imágenes servían al propósito de inspirar al lector a un ejercicio de reconocimiento positivo de esos personajes, no visibilizados socialmente. Es importante tener en cuenta que en las fotografías no aparecían personajes históricos ni extranjeros sino aquellos que se circunscribían a la realidad inmediata del contexto nacional colombiano, a partir de lo cual se buscaba batallar contra la idea de la inherente incapacidad del ciudadano negro para desempeñar cargos de manera responsable y, por elevación, reclamar por las oportunidades negadas por el Estado y la sociedad colombianos.

Este reclamo, por otra parte, era frecuente en los espacios de discusión sobre cuestiones raciales en esa época. Sin ir más lejos, es preciso recordar la encendida alocución del político colombiano Marino Viveros durante el Primer Congreso de la Cultura Negra de las Américas -realizado en Cali en 1977-, en la que a la problemática del bajo número de escaños de representación parlamentaria ocupados por ciudadanos negros le sumaba la preocupación por lo poderosa que resultaba la idea de "blanqueamiento" en el imaginario social de los grupos negros: "De los nueve curules [en el departamento del Cauca], ocho están en manos de los blancos. [...] esa es una forma de discriminación que no se ha corregido desde la época de la esclavitud hasta nuestros días y que en su lucha no vamos a encontrar nosotros como aliados a quienes están tratando de ocultar su propia identidad de negros" (cit. en Valero 124. Énfasis nuestro). Esta misma preocupación será abordada y analizada en numerosas ocasiones por Smith Córdoba, como veremos enseguida.

El esfuerzo de Smith Córdoba por mostrar un problema que él entendía no solo de modo social, sino epistemológico y abarcador de toda la sociedad colombiana, será el eje de las columnas "Aprender a ser Negro". No se puede ignorar que en los ańos setenta los movimientos políticos y culturales crecieron con la experiencia de lo que se llamó la generación Bandung y, más aún, con la inspiración de la Conferencia Tricontinental de la Habana en 1966, esto es, la conciencia de que el mundo colonial tal como se lo había conocido, con la fuerza del imperialismo europeo, sea física o epistemológicamente, iba en caída, o, al 
menos, tenía franca resistencia. De tal manera, estos espacios conformadores de la infraestructura intelectual negra también deben ser vistos a través de los lentes de una ideología global en términos de desalienación y resistencia descolonizadora, marco en el que se inscribía el racismo como huella de un colonialismo inacabado. De hecho, varios artículos de Smith Córdoba serán reacciones airadas contra el accionar de las potencias europeas y la propaganda afín a ellas y contra los gobiernos africanos. Al mismo tiempo, hará uso de una serie de conceptos propios de la época que se enmarcaban en esa lucha transnacional, aunque esta exceda lo racial: alienación, colonialismo, descolonización.

La problemática que Presencia Negra manifestaba reiteradamente en la columna "Aprender a ser Negro" se inscribía en ese trasfondo que, además, había encontrado un espacio de discusión abierta durante el Primer Congreso de la Cultura Negra de las Américas, al que Amir Smith Córdoba había asistido. Si algo había quedado claro allí, era que la batalla contra el neocolonialismo debía darse en el campo de la educación y de la política y, por lo tanto, las acciones debían ir dirigidas en ese sentido, si lo que se buscaba era la concientización del negro.

"Aprender a ser Negro" actuaba como una apelación directa a los ciudadanos negros colombianos, al tiempo que establecía con claridad el lineamiento ideológico de la publicación en su totalidad, que no se apartaba de lo discutido en general en la época con respecto a la situación de la gente negra: la transversalización clase-raza. La columna apuntaba contra quienes se distanciaban de su negritud al modificar su comportamiento y cambiar de medio social con fines de movilidad ascendente, lo cual se condenaba en términos de "blanqueamiento". Además, ponía en tela de juicio el alcance de este último y mostraba sus límites para un posicionamiento social cuando, al mismo tiempo, denunciaba la explotación socioeconómica emergente de la racialización de los sujetos negros. Esta última, muchas veces, facilitada por la ausencia de un autorreconocimiento en términos de concientización identitaria.

En ese juego de poderes, Smith Córdoba sumó otro componente que se acopló a este binomio compuesto por sujetos blanqueados y sujetos racializadores: el grupo a los que denominó "áskaris", apropiándose de un concepto utilizado por Aimé Césaire en su texto Cuaderno de retorno a un país natal. Con ese término, refería a ese negro que busca emular al blanco (Césaire 118-119), delegado por 
el colonialista para mantener un statu quo de la empresa colonial. A través de la figura del áskari, a la que el mismo sistema (neo)colonial le concede algún tipo de poder, el dominador ejerce un dominio prolongado en un nivel mental, es decir, en un nivel en el que la alienación funciona sin la necesidad de que el colonizador imponga su presencia física. El artículo "Los áskaris y el nacionalismo africano" se inscribía, así, como una ilustración de la "antropofagia" que señala Smith Córdoba entre pares negros. Además de reclamar por la autodeterminación de los africanos en cuanto a su derecho a solucionar sus propios problemas sin ningún tipo de intervencionismo por parte de Occidente, Smith Córdoba encontró una analogía entre el conflicto ugandés y el pensamiento de muchos colombianos negros quienes, como el áskari césaireano, eran traidores a su propia raza a cambio de beneficios o reconocimientos. Adaptando la retórica estereotipada que zoomorfiza al africano y sus descendientes, el chocoano, al mismo tiempo que dicotomizaba a la sociedad entre un "ellos" y un "nosotros" hiperreal, condenaba en estos términos a ese sector de la población negra:

La desconfianza entre nosotros [los negros] ha sido creada por ellos [los blancos], y es contra todos sus estereotipos que han instaurado como cultura que debemos darnos a la tarea de luchar a brazo partido e infatigablemente para rechazarlos. Es válido anotar que la tarea se torna difícil y complicada mientras haya entre nosotros quienes como amaestrados, sean capaces de responder como dóciles áskaris al servicio de sus domadores. Es triste contarlo pero es la verdad, las universidades de Europa están repletas de esta clase de gente, los que no sólo están dispuestos a vender, sino y lo que es peor, a comercializar la sangre de sus mismos hermanos. Lo uno es ser "civilizado" y lo otro es ser "salvaje", al contrario[,] se sienten muy agradecidos de saber que han sido invitados a bajarse del árbol para que en recompensa haga subir a sus hermanos (Smith Córdoba 5).

"Los áskaris y el nacionalismo africano", junto con otros artículos como "El Negro: su historia y su geografía", "Labor Integracionista", "Prejuicio: 'pequeño burgués" y "Lo cursi de la antropofagia política" actuaban como satélites de "Aprender a ser Negro", en tanto eran parte de una confluencia en el proyecto ontológico y teleológico de esa 
columna. Aunque los artículos se podían leer de forma independiente, es necesario advertir que hacían parte de una articulación mayor, que realizaba una labor ideológica (De la Fuente García 413).

Smith Córdoba, como persona que se definió a sí misma como negra, representaba a ese sector social y, como sujeto de discurso, hablaba en nombre de él. Al representar a instituciones como el CIDCUN y sus publicaciones - a las que constantemente se mencionaba en los artículos del periódico en carácter de referencias de autoridad-, no solo las instaló como indicadores de su conocimiento sobre el tema de "lo negro" en Colombia, sino que también él mismo se posicionaba como representante de las negritudes a través de su gestión como activista y fundador ${ }^{8}$. De allí que el periódico, más que un canal de información y comunicación, se asumió casi como portador de una misión, materializando el proyecto educativo-concientizador en la columna "Aprender a ser Negro":

Vale la pena aclarar que PRESENCIA NEGRA [énfasis en el original], órgano del "CENTRO PARA LA INVESTIGACIÓN DE LA CULTURA NEGRA EN COLOMBIA” [énfasis en el original], nunca será excluyente, por eso en la tarea de afirmar nuestra identidad, entramos a promover todos nuestros valores sin distingos, y en esta indiscriminada labor cabemos todos. Así que no podemos explicarnos ciertas preocupaciones de los que miran con desconfianza que por estas páginas se promuevan nombres de personas que nunca se han sentido negros, si nuestra tarea es de rescate precisamente, y aunque en última instancia no sea el color lo determinante, que se d[é] en el sentido práctico de las relaciones hay negros blancos como también se pueden dar los "blancos negros", esto sin entrar a descifrar causas. Es nuestro deber inculcar lo que significa, implica y representa nuestra verdadera identidad; es hacerles ver que no son blancos y que[,] como tal, no deben ni pueden ser ajenos a los problemas que debaten comunidades que no son precisamente las que están defendiendo en última instancia (Smith Córdoba 4. Énfasis nuestro).

8 En este sentido, Martín Agudelo señala que una característica sobresaliente del periódico es "el fuerte personalismo alrededor de su director [Smith Córdoba], que acapara a tal punto la publicación que a veces parece más una cruzada personal que una empresa colectiva” (100. Traducción propia). 
En esta perspectiva, Smith Córdoba percibía al negro "blanqueado" como producto de la alienación institucionalizada con fines de control del pensamiento. El periódico se constituía, así, en el puente para revelar este control cognitivo y romper con la despersonificación a la que había descendido el negro, como resultado de políticas públicas y cotidianas, prácticas y simbólicas. De acuerdo con ello, para que este proyecto se lleve a cabo se debían cumplir ciertos parámetros:

El negro debe y tiene que aprender a asumir su identidad sociocultural y como producto que es de una doble alienación, debe predisponerse a forzar unas nuevas situaciones en las que como protagonista de su búsqueda sabe que su labor no concluye con las limosnas y migajas, las que en ningún momento pueden ser cura para la enfermedad endémica y desafiante postración que vive él nacionalmente. Nos interesa[,] eso sí, entrar a analizar cómo y en qu[é] medida ciertas prebendas no se conviertan en paliativos que contribuyan de una u otra forma a agravar la enfermedad (Smith Córdoba 1).

Siempre y cuando el negro se apropiara de esa especificidad sociocultural de la que habla el autor, tendría "las oportunidades que se la han negado”. Para ello, Smith Córdoba juzgó crucial confrontar los aspectos psicológicamente debilitantes de una persona negra en una sociedad racista, desestigmatizando la negritud. La valorización de África tuvo su parte en este intento. De hecho, la lucha armada y anticolonial africana fue seguida por el diario Presencia Negra a través de artículos escritos por Smith Córdoba, donde se denunciaba el intervencionismo norteamericano y europeo, como en la invasión de Uganda a Tanzania. De allí que el chocoano creyera firmemente en que la autoconciencia que el negro debía adquirir se lograría mediante la instrumentalización de dos ejes principales: la educación, entendida como formación en la cultura negra, y la historia como espacio recordatorio del accionar negro en la historia del país. Ambos ejes, estudiados ya por García Rincón, el primero, y Valero y Ramos, el segundo (156-165), atravesaban la concepción del deber ser negro, y debían impactar políticamente en las comunidades negras colombianas, activando en ellas una conciencia de sí.

En ese orden, Smith Córdoba buscó, bajo el binomio negritud/ historia, revisar la disciplina histórica para liberar al negro de la carga esclavista, que había sido impuesta por los académicos colombianos en 
sus estudios (Valero y Ramos 148-149), y con esto motivar a una movilización en el campo de las reivindicaciones políticas que transformen realidades sociales, económicas y culturales, contemporáneas y futuras. Por lo tanto, fue frecuente en Presencia Negra la referencia a personajes negros de relevancia histórica, además de hechos donde se ponía de relieve su participación en la construcción del Estado-nación colombiano.

Paralelamente, y en función de los mismos objetivos, se concibió a la educación como vehículo indispensable para la movilización política de los colectivos negros (García Rincón). Por lo que "la participación en congresos y seminarios, más específicamente el Seminario sobre Formación y Capacitación de personal docente en Cultura Negra, fueron eslabones en la cadena de la transformación educativa que requería 'aprender a ser negro'” (Munar 100).

Antes de la fundación de Presencia Negra, Smith Córdoba había impulsado la organización, a través del CIDCUN, del Seminario sobre Formación y Capacitación de personal docente en Cultura Negra. Llevado a cabo entre el 12 y 14 de octubre de 1978 en la Universidad Pedagógica de Bogotá, constituyó uno de los componentes de la infraestructura intelectual negra que convergía en torno a la construcción de una identidad negra. La finalidad de este seminario era llegar a diseñar soluciones para la capacitación de docentes que fueran emisarios en la enseñanza de la cultura negra por todo el territorio nacional, aunque, como lo sugieren algunos investigadores y lo detallan otros, se "debió generar un verdadero ambiente de discusión” (Valero y Ramos 164) en el ámbito de los seminarios, en tanto los líderes de los mismos "representaron proyectos ideológicos de la negritud que rivaliza[ba]n entre sí" (Valderrama 234). Es evidente que para el CIDCUN y su director Amir Smith Córdoba, la educación cobraba un papel trascendental en el proyecto de "desavasallamiento cultural" contra la alienación del negro:

Este primer seminario, organizado por el "CENTRO PARA LA INVESTIGACIÓN DE LA CULTURA NEGRA EN COLOMBIA" [énfasis en el original], contó con la asistencia de destacados profesionales en las diferentes disciplinas del saber humano", los que dieron muestra en los amplios debates que se suscitaron durante el certamen, del interés y el empeño que los alentaba a investigar, divulgar y poder enseñar con propiedad lo que ha sido el verdadero aporte negro en la construcción de la identidad de los pueblos 
que aspiran, quieren y desean darse sus propias soluciones, lo que se podrá lograr sólo, en la medida en que se entre a afirmar una identidad encargada de hacer ver que la estructura social del negro es dueña de patrones que se han visto fortalecidos, gracias a sus creencias ancestrales que sirvieron no solo para aglutinarlos en muchos casos sino que merced a tan arraigadas convicciones dentro del marco de sus costumbres africanas, pudo poner resistencia al "sincretismo" abstracto de adoctrinamiento que le mostró occidente (la huida de sí mismo o blanqueamiento) (Smith Córdoba 1-2).

Consecuentemente, Amir Smith Córdoba planeó una serie de reformas educativas que propendieron a la enseñanza de la cultura negra en todos los niveles escolares. De la misma manera que se cuestionó cómo era llevada la disciplina histórica en Colombia, el chocoano propuso un cambio serio en todos los niveles educativos, que iría desde "la modificación y reestructuración de los textos académicos, literarios y todos aquellos materiales que cumplen una labor excluyente por la orientación que interiorizan" (Smith Córdoba 2), hasta la creación de una universidad que fortalezca y priorice las temáticas negras (Smith Córdoba 2). Esta centralización de la acción en proyectos educativos formales no fue un hecho casual ni aislado. Además de que habían sido motivo central de varias de las proposiciones y recomendaciones del Primer Congreso de la Cultura Negra de las Américas, publicadas en 1988, no se puede perder de vista el impulso que la Unesco venía sosteniendo con respecto a la investigación y la educación como caminos para el combate del racismo9. El periódico Presencia Negra tenía un vínculo especial con la entidad, en tanto recibía financiamiento como parte de una constante actividad de la Unesco en Colombia por esos años (Wade, "The Cultural Politics" 342). Definitivamente, la estrategia de "organizar un movimiento que inicie un proceso desalienador desde la infancia y exija una educación

9 La Unesco, desde el fin de la Segunda Guerra Mundial, movilizaba políticas contra el racismo en el mundo, asentadas en la investigación y la educación como una de las maneras de combatirlo. Llevaba adelante un programa "tendiente a fomentar el conocimiento de nociones científicas sobre la raza y a combatir así los prejuicios raciales" (Unesco, "Cuatro declaraciones" 7). Como parte de su accionar, respaldó luego financieramente al II Congreso de la Cultura Negra de las Américas ("Unesco y el II Congreso de la Cultura Negra a realizarse en Panamá”, Presencia negra (2) 5) 
secundaria y universitaria basada en la igualdad racial y en el derecho de todo ciudadano a afirmar su autenticidad étnica" (Fundación Colombiana de Investigaciones Folclóricas/Unesco 157) era clave en términos de los mecanismos para el autorreconocimiento.

Smith Córdoba, sin embargo, entendía que si bien los anteriores eran pasos urgentes en la lucha por una ciudadanía plena y un reconocimiento del componente negro de la colombianidad, el impacto en lo público debía llegar a otros espacios de la esfera social. Vio necesario interpelar a algunos periodistas no negros representativos de los medios hegemónicos con un contradiscurso que connotaba un proceso de reconfiguración del Estado-nación y sus políticas a partir del cuestionamiento del orden racial en el país. Sin embargo, esto no se desarraiga de la anterior estrategia, en tanto las respuestas a algunos columnistas suponían una manera alternativa de "enseñar a ser negro", en la medida en que se enfrentaba a discursos canalizadores de prejuicios raciales, interiorizados tanto por el blanco-mestizo como por el negro también.

Aunque Smith Córdoba hizo referencia de manera general, en algunos artículos, al blanco-andino o blanco-mestizo, se dirigió en especial a lo que él denominaba "pequeños burgueses", apropiándose del concepto con el que Aimé Césaire había clasificado a aquellos reproductores ideológicos del colonialismo. Decía Césaire:

No se puede decir que el pequeño burgués no haya leído nada. Él, por el contrario, ha leído todo, ha devorado todo.

$\mathrm{Su}$ cerebro funciona únicamente a la manera de algunos aparatos digestivos de tipo elemental. Él filtra. Y el filtro no deja pasar sino lo que puede alimentar la torpeza de la buena conciencia burguesa (25).

El trabajo del pequeño burgués - categoría en la que Césaire, ubicado en el contexto de la posguerra, reunía a científicos, pensadores sociales, escritores, religiosos, entre otros- es el de asimilar y sostener discursivamente la ideología de la clase dominante a través de los aparatos que tiene a su disposición. De tal modo, condenaba a los portadores culturales e intelectuales del poder colonialista que filtraban y acomodaban la información haciéndola pasar por argumentos sagaces. 
A partir de las influencias que recibió del marxismo y el Discurso sobre el colonialismo de Césaire, Smith Córdoba se apropió del concepto en un artículo aparecido en la edición dominical de El Tiempo, titulado "Razones y sinrazones de 'el pequeño burgués" (9), que es, a su vez, una respuesta al artículo “¿Qué es un pequeño burgués?”, de Antonio Panesso Robledo (8). Para Smith Córdoba, la figura del pequeño burgués sostenedor del statu quo se configuraba como un activo participante en la conformación del sentido común. Devenía, entonces, en un engranaje necesario para la asimilación de la blancura como posición estructural de privilegio. El chocoano ubicó a determinado sector de la prensa como ese intermediario pedagogizador:

Precisamente, una cosa se hace necesario aclarar, que al no caber todos en la danza económica del poder, originó la clase media, que es la que generalmente ha dado los ideólogos del sistema. Como respuesta a ello [a no participar de la danza económica], se muestran [la clase media] como consuetudinarios asimilados en el sentido íntegro del carácter servil (pequeño-burgués) "contento" ("Razones y sinrazones" 9).

Smith Córdoba encontró en cierto sector del periodismo colombiano una clase letrada representativa de ese servilismo pequeñoburgués resaltado en su texto, reproductora de una ideología neocolonial, asimilada por los valores que detentaban las clases dominantes con miras a mantener la jerarquización social y racial. De hecho, la columna que el periodista satírico Lucas Caballero Calderón (Klim) publicó en El Espectador, "Uganda, Tanzania y Colombia" (3a), provocó una encendida respuesta por parte de Smith Córdoba a través el artículo "Lo Klim no quita lo Caballero" (Smith Córdoba 6).

"Uganda, Tanzania y Colombia" tomó como pretexto el conflicto bélico entre las dos naciones africanas y la invasión a la república tanzana el 3 de octubre de 1978 por Idi Amín Dadá, presidente de Uganda, para referirse en realidad, por asimilación de contextos y personas, a la actualidad política colombiana. Al reiterar indiscriminadamente categorizaciones como "simiocracias", "cuadrumanos", "salvajes", "simios", "antropoides", "antropófagos" y otras similares para referirse a Uganda y a su presidente, Klim no estaba haciendo otra cosa que preparando el terreno para llegar al contexto colombiano e ironizar sobre la con- 
ducta oficial. Acción mediada, en esta oportunidad, por la referencia pretendidamente jocosa al color de piel del ministro de Agricultura de entonces, Germán Bula Hoyos, lo que lo convertía, bajo la pluma caricaturizadora de Klim, en un salvaje primate, como se muestra en el siguiente fragmento:

¿Por qué, decide [la Sociedad Protectora de Animales], mantener en cautiverio a este vigoroso cuadraumano [Idi Amín Dadá], si muchos de sus congéneres disfrutan de su libertad en las selvas de África y algunos de ellos, como Edson Arantes do Nascimento Pelé, se destacan en el deporte o son nombrados miembros del gabinete ejecutivo como Bula Hoyos en Colombia? (El Espectador

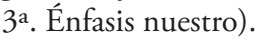

El temor nuestro es que el mal ejemplo [Tanzania y Uganda] cunda, ahora cuando se están presentando enfrentamientos entre los miembros del propio gabinete ejecutivo, verbigracia entre los ministros de Hacienda y Agricultura. Y que este, sintiendo hervir en su sangre el ardor secular de los grandes antropoides africanos, se suba a un árbol a lanzarle cocos a García Parra ${ }^{10}$ hasta matarlo (El Espectador 3a).

Smith Córdoba entendía que en Colombia se proyectaba lo que Van Dijk considera racismo cotidiano, esto es, prácticas sociales usuales que operan en lo económico y sociocultural a través de mecanismos de subordinación y marginación: "Dentro de los patrones de domino del racismo cotidiano en Latinoamérica el aspecto 'raza' y la cultura suelen entrelazarse y convertirse en una sola cosa, es decir, en que son 'diferentes' de 'nosotros'” (102). Uno de los aparatos del poder canalizador de ese racismo cotidiano, devenido en sentido común, era el constituido por los medios de comunicación, que preformulaban categorías sociales de diferencia, criterios de superioridad, pautas, es decir, todo el andamiaje necesario para la legitimación del racismo. Precisamente, en la respuesta a "Uganda, Tanzania y Colombia", al titularla "Lo Klim no quita lo Caballero", el autor pone de manifiesto que ve en el artículo un indicador de la ideología de las clases influyentes bogotanas entre las que se había criado Caballero Calderón, y que quedaba en evidencia: aunque se enmascare bajo el seudónimo de Klim y fuera reconocido por su pluma ácida y su constante 
ironización sobre las irregularidades del gobierno de Alfonso López Michelsen (1974-1978) y sobre la caricaturización de sus miembros, su ideología de clase lo superaba.

En su artículo, Smith Córdoba asumió que el sentido humorístico de la columna de Caballero Calderón no solo daba cuenta de la distribución asimétrica de poder en tanto decía quién se podía reír de quién. Además de ignorar la sensibilidad del grupo de referencia, sostenía la diseminación y el refuerzo de una imagen social negativa de los ciudadanos negros en un periódico de gran tiraje a nivel nacional, cuyos lectores seguramente compartirían la línea ideológica de la columna. En este sentido, Smith Córdoba interpelaba la ideología del columnista:

Y menos podemos tolerar que alguien mordido por sus bajas pasiones, con lo que pretende acariciar, estimular y endulzar, no solo el oído, sino la intención racista de los que como él, sienten que por sus venas corre la imponderable y "pura" sangre azul de sus ancestros espańoles. Olvidando de paso la mezcla de los pueblos y lo que ellos representaban cuando invadieron y destruyeron gran parte de la dignidad americana ("Lo Klim no quita" 3a).

Dejando de lado el continuo trasfondo de crítica política con el que cargaban las columnas de Caballero Calderón, lo que movilizó a Smith Córdoba fue el sesgo racista de "Uganda, Tanzania y Colombia" y para su rechazo se apoyó en el mismo recurso de zoomorfización del que se había valido Klim, pero invirtiendo el destinatario, de tal manera que la bipolaridad ideológica civilizados/bárbaros revirtiera sus agentes y, al mismo tiempo, permitiera lo que el chocoano buscaba: desenmascarar el proceso de alienación "del blanco" colombiano (el pequeño burgués de Césaire), representado en Klim. En un fragmento en el que en el inicio resuena el Césaire del Discurso del colonialismo, Smith Córdoba resumía lo que consideraba la doble alienación del periodista:

Su mal fundada versión acerca del "antropofagismo" de Amín, [i] es o no mucho más aceptable que los genocidios abiertos o soterrados que en nombre del humanismo, la democracia y de la "civilización" han cometido los "grandes" del sistema occidental imperialista? [i] Negará acaso que se identifica con quienes aún piensan que el blanco nació para domesticar a otros pueblos? [i] 
O tratará de ignorar por el descuido miope de sus enajenaciones que[,] para el blanco, todo lo que no es blanco es negro, y que en su afán cursi de sentirse blanco, sus cálculos pueden fallar y lo de "sangre azulada" que corre por sus venas a lo mejor no le sería útil, si por desgracia el fascismo triunfara en América[?] ("Lo Klim no quita" 6a).

Para Smith Córdoba, la razón por la cual era posible la publicación de un artículo del calibre del de Klim en Colombia se sustentaba en que el racismo operaba en el discurso de manera implícita de forma cotidiana. Es por ello que lo que leyó entre líneas Smith Córdoba fue que Caballero Calderón, con el lenguaje humorístico como herramienta aparentemente inocente, terminaba justificando los discursos históricos del país, tanto políticos como científicos en los que estaban inscritas la miseria y la marginalización de las costas Caribe y Pacífica colombianas. En los conceptos que contenía ese lenguaje se sustentaba la no inversión estatal ni privada en los sectores negros de la población ${ }^{11}$. He ahí la peligrosidad de seguir sosteniendo la retórica del discurso racista: avalar mediante el discurso una práctica material y real de discriminación.

Smith Córdoba cierra su defensa vaticinando "un proceso que se encargará de hacer ver en la práctica lo que implica en sí nuestra verdadera identidad sociocultural" ("Lo Klim no quita" 6) a pesar de los "segregacionistas" ("Lo Klim no quita" 3) que, como Klim o el periodista antioqueño Panesso Robledo (Pangloss), se hallaban anclados en las estructuras sociales.

El artículo de Pangloss, "Los Negritos en Color", publicado en El Espectador en 1978, fue el disparador para el desarrollo de otra columna-respuesta en Presencia Negra, a través de la cual Smith Córdoba dio cuenta de varias claves que blancos y negros debían interiorizar con vistas a un cambio social real en términos de orden racial de la nación.

Pangloss escribió "Los Negritos en Color" en el contexto de la aparición del libro Raíces, del afronorteamericano Alexander Murray Palmer Haley. Raices es la historia de Kunta Kinte, natural de Gambia, secuestrado durante el siglo XVIII y convertido en esclavo en Estados Unidos por los ingleses. La importancia de este libro radicaba en el contexto en el que se enmarcaba su publicación, agosto de 1976, que

11 Ver “¿Y para el Chocó qué?” (Smith Córdoba 1-5). 
coincidía con la lucha por los derechos de los negros en Estados Unidos. Raíces pasó a convertirse en una fuente de la narrativa de la lucha de los colectivos afroamericanos, en primera instancia, pero rápidamente, sobre todo por su posterior adaptación a la televisión que llegó una audiencia mayor, significó una reivindicación de la cultura negroafricana en América, además de denunciar y desentrañar el racismo heredado del sistema colonial y la esclavitud.

En "Los Negritos en Color", Pangloss examinaba la acogida de Raices, tanto a nivel literario como en su adaptación a la televisión, poniendo en tela de juicio la campańa publicitaria de Haley por diferentes países del mundo, lo cual era visto con cierta sospecha de inautenticidad por el antioqueño por el beneficio económico que representaba para su autor. Expresaba Pangloss que:

Cuando este género [novela histórica] se combina con problemas sociales o políticos, como es el caso de los negros de Estados Unidos, la fórmula no puede fallar nunca. Y no le ha fallado a Alex Haley, quien está ahora percibiendo los derechos del libro y los derechos de la película. Con una sola semana de sus ganancias actuales habría podido rescatar a Kunta Kinte y a toda su familia. Lo cual plantea otro problema: ‘̧a negros como Alex Haley les habría ido mejor quedándose en Ghana o en Gambia o en Guinea, más bien que trasladarse a Estados Unidos? Es esta, por supuesto, una pregunta de mal gusto que no debe hacer nunca una persona de buenas maneras (11).

Smith Córdoba responde a Pangloss con "La patria boba y sus orientadores" (3), donde cataloga al periodista como "uno de los 'ideólogos' más peligrosos entre los que aún quieren seguir pintando y cultivando la patria boba de antaño" (ibid.), lo que lo incluye como ejemplo del pequeño burgués referido más arriba: "Se dibuja a todas luces su racismo anticuado, pero domesticador en sus intenciones" (ibid.). Smith Córdoba leía en ello la intención de desestimar la voz de los negros como portadora de su propia historia: "No veo por qué ese empeño desmesurado en seguir ocultando una historia que debe encontrar un nuevo y más adecuado tratamiento por o de quienes deben pasar a decidir su propia 'suerte' y 'destino'” (3). 
Pero su intención no era solo crítica sino también propositiva (Arévalo 23-24), en tanto, a tono con el clima de la época en Colombia en materia de agrupaciones en pro de los derechos de los negros, dejaba en claro la capacidad de reacción de los intelectuales negros frente a ataques del tipo que hemos visto:

Nuestro pasado histórico es y será el encargado de vivificar un presente dispuesto a empujarnos a un futuro reivindicador, lo que constituirá necesariamente un arma para la liberación en todos los órdenes y en todos los sentidos; y nuestra reacción será la de organizarnos, para exigir de esa manera una mejor y más adecuada participación (Smith Córdoba, "La patria boba" 3).

En este apartado del artículo, Smith Córdoba resumía los puntos clave de su proyecto cultural y político. Por un lado, la historia debía ser el eje principal que impulsara las reivindicaciones del negro tanto en el presente como en el futuro, actuando, así, como pedagogía de la desalienación cultural, política y social. Por otro lado, mediante esta instrumentalización del discurso histórico se podría llegar a una acción que impulse a la participación política. De tal modo, la historia, en Smith Córdoba, homogeniza a un grupo social con miras a ser parte integral de la construcción de la nación a través de sujetos reeducados en la negritud, es decir, en el reconocimiento de sí mismos. Pero ello, para que la acción encauzada sea integral, debía funcionar en una doble vía, esto es, con el reconocimiento del negro como ciudadano en igualdad de condiciones y derechos que el resto de la población nacional.

\section{Conclusiones}

Siguiendo al historiador George Reid Andrews, podemos decir que el proyecto de Amir Smith Córdoba hizo parte del "ennegrecimiento" que tuvo Afrolatinoamérica en la década de los setenta, con la creación de movimientos sociales y "espacios de sociabilidad y experiencias asociativas de la gente negra" (Munar Espinosa 105). En este sentido, el proyecto de desavasallamiento cultural del chocoano procuró crear un espacio alternativo desde donde se denunciaron los problemas de racismo y discriminación contra las comunidades negras colombianas. 
Con herramientas conceptuales como la negritud y la alienación, Smith Córdoba buscó también desenmascarar el racismo cotidiano en los discursos de algunos periodistas colombianos que, inscritos en una estructura ideológica colonial, sostenían desde los medios de comunicación un racismo antinegro.

Aunque la denuncia del racismo es uno de los tópicos principales en el proyecto del sociólogo chocoano, el autorreconocimiento del negro se instituye como la parte fundamental de su proyecto, es decir, la toma de conciencia, la autoconciencia de la historia, los valores culturales, la afirmación de un ancestro. Todo ello para construir una identidad negra que repercuta en pro del reclamo por los derechos que históricamente se les habían negado a estas comunidades:

[E]l negro debe darse a la tarea de reclamar con prestante solvencia el puesto que de verdad le corresponde, que no es ni lo será nunca una dádiva, es cobrar su sitial de acuerdo a lo que ha significado su aporte a la vida nacional, lo que entramos y debemos entrar a vitalizar como una acción de legítimo derecho (Smith Córdoba 5).

A través del CIDCUN, con "los manuales" (Smith Córdoba, Cultura 26) Negritud y Presencia Negra, Smith Córdoba buscó combatir "la sobreimposición cultural de Occidente" ("La patria boba" 3), leída como ideologías coloniales ancladas en el imaginario colombiano. En ese sentido, dichas plataformas debían funcionar como pedagogizadoras a través de las categorías de negritud y desalienación, pero no solo para el negro sino también para el resto de los colombianos, con el fin de construir reivindicaciones de las comunidades negras a través de la historia, para el presente y el futuro.

Bibliografía

Andrews, George Reid. Afro-Latinoamérica 1800-2000. Madrid, Iberoamericana-Vervuert, 2007.

Arboleda Quiñonez, Santiago. Le han florecido nuevas estrellas al cielo: suficiencias intimas y clandestinización del pensamiento afrocolombiano. Tesis para optar al grado de Doctor en Estudios Latinoamericanos, 
Cali, Universidad Andina Simón Bolívar, 2011. Web. Disponible en: https://cutt.ly/4oCxHMb.

Arévalo Méndez, Julio. Amir Smith Córdoba: del blanqueamiento a la negritud. Tesis para optar al grado de Magíster en Estudios Culturales, Bogotá, Pontificia Universidad Javeriana, 2015. Web. Disponible en: https://cutt.ly/SoCvEXO

CÉsaire, Aimé. Cuaderno de un retorno al país natal. Ciudad de México, Era, 1969.

. Discurso sobre el colonialismo. Madrid, Akal, 2006.

De la Fuente García, Mario. "El Análisis Crítico del Discurso: una nueva perspectiva”. Contextos, vol. 19/20, №37/40, 2002, pp. 407414. Web. Disponible en: https://buleria.unileon.es/handle/10612/699

Fanon, Frantz. Los condenados de la tierra. Ciudad de México, Fondo de Cultura Económica, 1963.

. Piel negra, máscaras blancas, Madrid, Akal, 2009.

Fundación Colombiana de Investigaciones Folclóricas/Unesco. Primer Congreso de la Cultura Negra de las Américas. Cali, Fundación Colombiana de Investigaciones Folklóricas/Unesco, 1988.

Gutiérrez, Idelfonso. Historia del negro en Colombia. Bogotá, Nueva América, 1980.

García Rincón, Jorge. "El racismo en Colombia: la visión de Amir Smith Córdoba”. Revista del Cisen Tramas/Maepova, N7, 2019, pp. 123-138. Web. Disponible en: http://ppct.caicyt.gov.ar/index. $\mathrm{php} / \mathrm{cisen} /$ index

Klim. "Uganda, Tanzania y Colombia”. El Espectador, 8 de noviembre de 1978, p. 3 a.

Martin Agudelo, Laura. Apprendre à être noir dans une nation métisse. La revue Presencia Negra et les mouvements noirs en Colombie dans les années 1980. Tesis para optar al grado de Magíster en Historia. París, Université Sorbonne Nouvelle-Paris 3-Institut des Hautes Études de l'Amérique latine, 2019. Web. Disponible en: https:// dumas.ccsd.cnrs.fr/dumas-02184929/document

Munar Espinosa, Lery. "Reivindicación de la 'cultura negra' y la denuncia de discriminación racial en la experiencia del Centro de Estudios Afrocolombianos y el Centro para la Investigación de la 
Cultura Negra (CIDCUN)”. Visitas al Patio vol. 14, Nº 1, 2020, pp. 85-106. Web. Disponible en: https://doi.org/10.32997/RVP-vol.14num.1-2020-2605

Panesso Robledo, Antonio (Pangloss). “¿Qué es un pequeño burgués?”. Magazin Dominical de El Espectador, 24 de noviembre de 1974, p. 8. . "Los Negritos en Color". Magazin Dominical de El Espectador, 19 de febrero de 1978, p. 11.

Pisano, Pietro. "Movilidad social e identidad 'negra' en la segunda mitad del siglo XX". Anuario colombiano de historia social y de la cultura, $\mathrm{N}^{\circ}$ 41-42, 2014, pp. 179-199. Web. Disponible en: https:// doi.org/10.15446/achsc.v41n1.44854

Pita González, Alexandra. "Las revistas culturales como soportes materiales, prácticas sociales y espacios de sociabilidad". Almacenes de un tiempo en fuga: Revistas culturales en la modernidad hispánica, Hanno Ehrlicher y Nanette Rissler-Pipka (eds.), Augsburgo, Universität Augsburg, 2014, Web. Disponible en: https://www.revistas-culturales. de/es/buchseite/alexandra-pita-gonz\%C3\%A1lez-las-revistas-culturales-como-soportes-materiales-pr $\% \mathrm{C} 3 \% \mathrm{~A} 1$ cticas

Realpe Borja, Marco. "Presencia Negra". Presencia Negra, Nº I, 1979, p. 5. CIDCUN.

Smith Córdoba, Amir, "Razones y sinrazones del 'pequeño burgués". Lecturas Dominicales de El Tiempo, 26 de enero de 1975, p. 9. . "Aprender a ser Negro", Presencia Negra, (I), 1979, pp. 1-6, CIDCUN.

. "Aprender a ser Negro". Presencia Negra, No II, 1979, pp. 1-6. CIDCUN.

. "Aprender a ser Negro". Presencia Negra, N III, 1979, pp. 1-5. CIDCUN.

. "Conclusiones del 'I Seminario sobre formación y capacitación de personal docente en Cultura Negra"'. Presencia Negra, $\mathrm{N}^{\circ}$ I, 1979, pp. 1-2. CIDCUN.

"Conclusiones del 'I Seminario sobre formación y capacitación de personal docente en Cultura Negra”. Presencia Negra, $\mathrm{N}^{\circ}$ II, 1979, pp. 1-2. CIDCUN. 
. "Conclusiones del 'I Seminario sobre formación y capacitación de personal docente en Cultura Negra”'. Presencia Negra, $\mathrm{N}^{\circ}$ III, 1979, pp. 1-2. CIDCUN.

"El Negro: su historia y su geografía". Presencia Negra, $\mathrm{N}^{\circ}$ II, 1979, pp. 3-12. CIDCUN.

. "La Patria boba y sus orientadores". Presencia Negra, N III, 1979, p. 3. CIDCUN.

4. CIDCUN.

. "Labor Integracionista". Presencia Negra, N II, 1979, p. . "Lo cursi de la antropofagia política". Presencia Negra, $\mathrm{N}^{\circ}$ II, 1979, p. 5. CIDCUN.

. "Lo Klim no quita lo Caballero". Presencia Negra, No I, 1979, pp. 3-6. CIDCUN.

. "Los áskaris y el nacionalismo africano". Presencia Negra, No III, 1979, p. 5, CIDCUN.

. "Prejuicio: 'pequeño burgués", Presencia Negra, No III, 1979, p. 4. CIDCUN.

1-5. CIDCUN.

"Y para el Chocó qué?". Presencia Negra, Nº I, 1979, pp.

. Cultura Negra y avasallamiento cultural. Bogotá, MAP, 1980.

Unesco. "Cuatro declaraciones sobre la cuestión racial". París, Unesco, 1969.

Valero, Silvia. "Los Negros se toman la palabra". Primer Congreso de la Cultura Negra de las Américas: debates al interior de las comisiones y plenarias. Bogotá y Cartagena, Universidad Javeriana/Universidad de Cartagena/Centro de Estudios Afrodescendientes, 2020.

Valero, Silvia y Erick Ramos. "Amir Smith Córdoba y la revista Negritud (1976): contrapuntos por el lugar del negro en la historia”. Memorias. Revista Digital de Historia y Arqueologia del Caribe Colombiano, N4, 2020, pp. 146-170. Web. Disponible en: https:// cutt.ly/szQlCVb

Valderrama, Carlos. "La diferancia cultural negra en Colombia. Contrapúblicos afrocolombianos". Revista CS, N²9, 2019, pp. 209242. Web. Disponible en: https://doi.org/10.18046/recs.i29.3631 
Van Dijk, Teun. Dominación étnica y racismo discursivo en España y Latinoamérica. Barcelona, Gedisa, 2003.

Wade, Peter. "The Cultural Politics of Blackness in Colombia". American Ethnologist vol. 22, N², 1995, pp. 341-357. Web. Disponible en: http://www.jstor.org/stable/646706

Wade, Peter. "Defining Blackness in Colombia". Journal de la Société des Américanistes, №95, 2009, pp. 165-184. Web. Disponible en: https://doi.org/10.4000/jsa.10783

Recepción: 26-09-20

Aceptación: 11-01-21 